\title{
A Case of Acute Intestinal Obstruction Caused by Remnant Fallopian Tube after Abdominal Hysterectomy
}

\author{
Sinuvasan Karunanithy ${ }^{1}$, Rahul Raj Chennam Lakshmikumar², Sampathkumar Poral ${ }^{3}$, Marenika Manisekaran ${ }^{4}$ \\ 1,2,3,4 Department of General Surgery, Pondicherry Institute of Medical Sciences, Kalapet, Puducherry, India.
}

\section{INTRODUCTION}

Abdominal hysterectomy is one of the common procedures done in India most commonly for dysfunctional uterine bleeding. Bowel obstruction occurs when the normal flow of intraluminal contents is interrupted. It may be caused by the pathology affecting the peristalsis, or mechanical. Bowel obstruction is usually managed with prompt surgical intervention.

In this case report, we discuss an unusual presentation of intestinal obstruction which was caused by adhesions around the remnant fallopian tube.

\section{PRESENTATION OF CASE}

A 56-year-old female presented to casualty with complaints of abdominal pain for 3 days and vomiting for 2 days. She was a known case of hypertension, diabetes mellitus, stable angina for 7 years and was on medication. Is past h/o abdominal hysterectomy done 20 years before due to? Dysfunctional uterine bleeding (medical records unavailable). On examination she was restless, tachypnoeic, tachycardic, hypertensive, uncontrolled blood sugars, and feculent drain from the nasogastric tube. Abdominal examination showed low midline scar, distension with tenderness present at umbilical, hypogastric and both iliac regions, showed guarding and rigidity at the umbilical, right lumbar and iliac regions and sluggish bowel sounds. X-ray erect and supine abdomen showed dilated bowel loops and multiple air-fluid levels.
Corresponding Author: Dr. Sinuvasan Karunanithy, Postgraduate,

Department of General Surgery,

Pondicherry Institute of Medical Sciences, Kalapet, Puducherry, India.

E-mail: k.sinuvasanganesh@gmail.com

DOI: $10.14260 /$ jemds/2022/60

How to Cite This Article: Karunanithy $S$, Lakshmikumar RRC, Poral $S$, et al. A case of acute intestinal obstruction caused by remnant fallopian tube after abdominal hysterectomy. J Evolution Med Dent Sci 2022;11(01):315317, DOI: 10.14260/jemds/2022/60

Submission 14-12-2021,

Peer Review 21-12-2021,

Acceptance 20-01-2022,

Published 31-01-2022.

Copyright (C) 2022 Sinuvasan Karunanithy et al. This is an open access article distributed under Creative Commons Attribution License [Attribution 4.0 International (CC BY 4.0)] 


\section{CLINICAL DIAGNOSIS}

Diagnosis of intestinal obstruction was confirmed clinically and radiologically. She underwent an emergency laparotomy. A midline incision was done and the abdomen opened from the symphysis pubis to the supra umbilical region. Intraoperatively, dilated small bowels were noted with adhesions along the remnant fallopian tube which was encircling the ileal segment causing an obstruction. Adhesions and remnant fallopian tubes were removed. The viability of the bowel was checked by stimulation, $100 \%$ oxygen mesenteric pulsation and visible peristalsis were seen. No other sites obstruction or perforation was noted. No bowel segment was resected. A thorough wash was given. Drains were kept at the subhepatic and pelvic regions and the abdomen closed in layers.
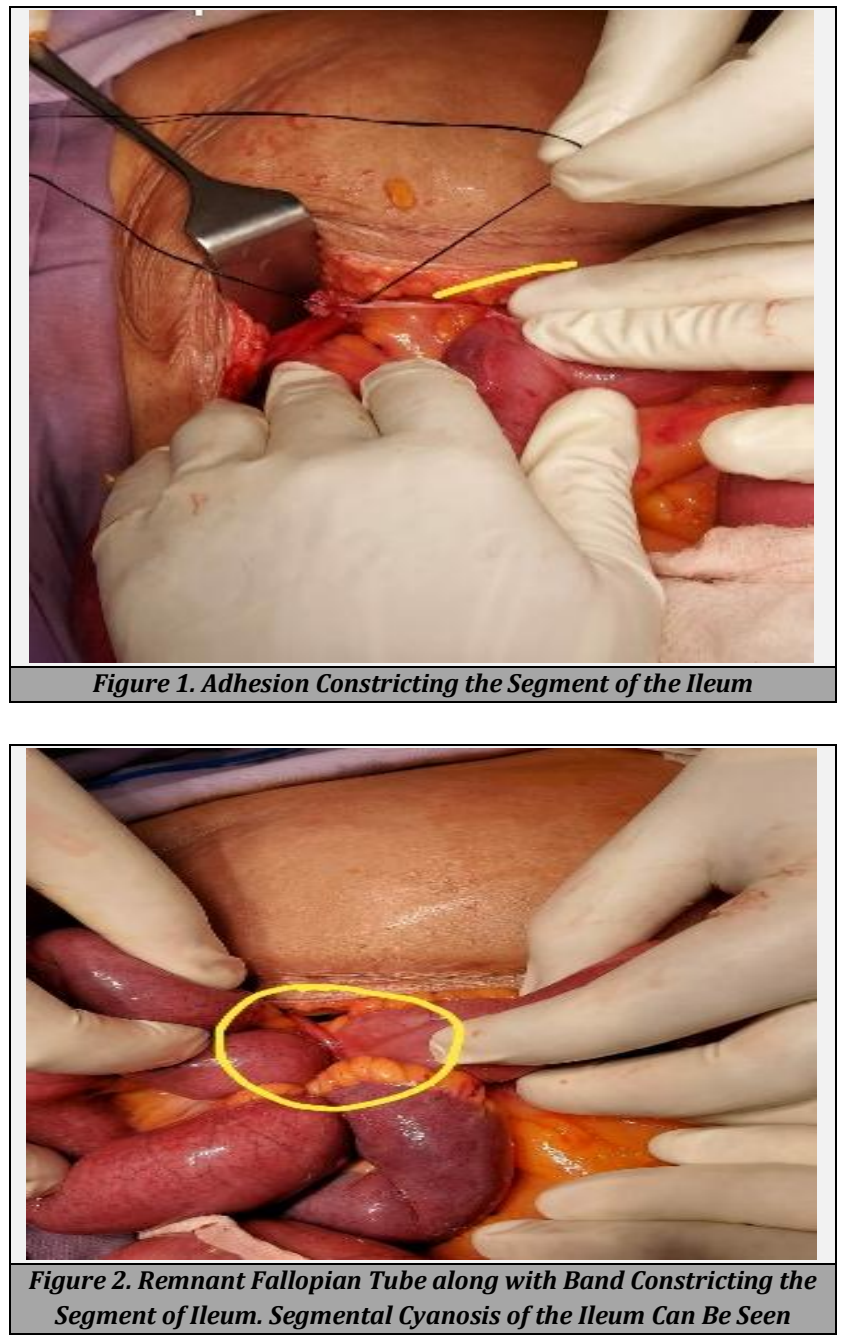

Postoperatively she was started on oral sips of fluids on POD -4 which was stopped due to abdominal distension. She was serially monitored for vitals, abdominal girth and subsiding of symptoms. She was then restarted with gradual sips of oral fluids on POD - 7 and a soft diet on the following day. X-ray abdomen erect and supine showed air-fluid level hence therapeutic oral gastrografin was given along with inj. metoclopramide due to gastroparesis. Hypokalemia was strictly corrected and a potassium-rich diet was given. Subsequently her abdominal complaints. Wound gapping with serous discharge secondary to fat necrosis was noted.
Wound swab from the operated wound showed the resistant strain of Acinetobacter baumannii, hence alternate sutures were removed and the daily dressing was done. Secondary suturing was done after the wound was healthy. She was then discharged with advice to follow up.

\section{DISCUSSION OF MANAGEMENT}

Abdominal surgery for various reasons can predispose to adhesion formation thereby resulting in acute or subacute intestinal obstruction. Adhesions are the abnormal fibrous bands of the normal healing process in the injured peritoneum due to disruption of the fibrinolytic system connecting the surfaces of the organ (which are usually separated).1,2 Small bowel obstruction accounts for 12-16\% of emergency surgical admissions ${ }^{3}$ and $20 \%$ of emergency surgical procedures. ${ }^{4}$ Apart from the above-mentioned sequalae, other complications that are commonly encountered due to adhesions are chronic abdominal pain, infertility, cost burden, difficulties at operation etc. ${ }^{5}$ Initial management should focus on correcting fluid and electrolyte imbalances and if clinical condition necessitates it should be proceeded with immediate surgical intervention. ${ }^{6}$ Some of the methods that can prevent postoperative adhesion formation are meticulous surgical techniques such as using a less invasive technique like laparoscopy, minimizing the tissue handling such as limiting of packing, crushing and manipulation of tissues. Barrier agents such as Polytetrafluoroethylene (GoreTex), sodium hyaluronate/ carboxymethylcellulose (Serpafilm) can be recommended that are shown to decrease adhesion formation in gynaecological surgery compared to non-use of any barrier agents. $^{7}$ The possibility of mechanical intestinal obstruction by fallopian tube should be kept in mind in both unoperated women and in patients who underwent hysterectomy with the leftover fallopian tube. A similar case of intestinal obstruction caused by entrapment of fallopian tube in the broad ligament defect was reported by Cameroon et al. ${ }^{8}$ In this study, both adhesion band formation and mechanical obstruction by remnant fallopian tube both contribute to intestinal obstruction. The timely surgical intervention had avoided the need for resection of the bowel. Surgeons need to adopt an adhesion reduction strategy for better surgical outcomes.

Financial or other competing interests: None.

Disclosure forms provided by the authors are available with the full text of this article at jemds.com.

\section{REFERENCES}

[1] Vrijland WW, Jeekel J, Van Geldorp HJ, et al. Abdominal adhesions: intestinal obstruction, pain, and infertility. Surg Endosc 2003;17(7):1017-22.

[2] Reddy SRR, Cappell MS. A systematic review of the clinical presentation, diagnosis, and treatment of small bowel obstruction. Curr Gastroenterol Rep 2017;19(6):28.

[3] Maung AA, Johnson DC, Piper GL, et al. Evaluation and management of small-bowel obstruction: an eastern 
association for the surgery of trauma practice management guideline. J Trauma Acute Care Surg 2012;73(5 Suppl 4):362-69.

[4] Millet I, Ruyer A, Alili C, et al. Adhesive small-bowel obstruction: value of CT in identifying findings associated with the effectiveness of nonsurgical treatment. Radiology 2014;273(2):425-32.

[5] Ten Broek RPG, Issa Y, van Santbrink EJ, et al. Burden of adhesions in abdominal and pelvic surgery: systematic review and met-analysis. BMJ 2013;347:f5588.
[6] Tong JWV, Lingam P, Shelat VG. Adhesive small bowel obstruction - an update. Acute Med Surg 2020;7(1):e587.

[7] Robertson D, Lefebvre G, Leyland N, et al. Adhesion prevention in gynaecological surgery. J Obstet Gynaecol Can 2012;32(6):598-602.

[8] Cameron M, Janakan G, Birch D, et al. A closed loop obstruction caused by entrapment of the fallopian tube and herniation through the broad ligament. Int J Surg Case Rep 2015;12:57-9. 